\title{
BMJ Open Mutation screening for thalassaemia in the Jino ethnic minority population of Yunnan Province, Southwest China
}

\author{
Shiyun Wang, ${ }^{1}$ Rong Zhang, ${ }^{1}$ Guangxin Xiang, ${ }^{2}$ Yang Li, ${ }^{2}$ Xuhong Hou, ${ }^{1}$ \\ Fusong Jiang, ${ }^{1}$ Feng Jiang, ${ }^{1}$ Cheng Hu, ${ }^{1}$ Weiping Jia ${ }^{1}$
}

To cite: Wang S, Zhang R, Xiang G, et al. Mutation screening for thalassaemia in the Jino ethnic minority population of Yunnan Province, Southwest China. BMJ Open 2015;5:e010047. doi:10.1136/bmjopen-2015010047

- Prepublication history for this paper is available online. To view these files please visit the journal online (http://dx.doi.org/10.1136/ bmjopen-2015-010047)

Received 20 September 2015 Revised 4 November 2015 Accepted 2 December 2015

\section{CrossMark}

${ }^{1}$ Shanghai Diabetes Institute Shanghai Key Laboratory of Diabetes Mellitus, Shanghai Clinical Center for Diabetes, Shanghai Jiao Tong University Affiliated Sixth People's Hospital, Shanghai, People's Republic of China

${ }^{2}$ National Engineering Research Center for Beijing Biochip Technology, Beijing, People's Republic of China

Correspondence to Professor Weiping Jia; wpjia@sjtu.edu.cn

\section{ABSTRACT}

Objectives: This study aimed to detect $\alpha$ - and $\beta$ thalassaemia mutations in the Jino ethnic minority population of Yunnan Province, Southwest China.

Design: A total of 1613 Jino adults were continuously recruited from February 2012 to April 2012. Fasting venous blood samples were obtained to determine haematological variables. Haemoglobin analysis was conducted using high-performance liquid chromatography. Participants with hypochromic microcytic anaemia or positive haemoglobin analysis profiles were confirmed by $\alpha$ - and $\beta$-globin genetic testing, including DNA microarray analysis, direct sequencing methods and multiplex gap-PCR assays. Setting: Shanghai Diabetes Institute, Shanghai Key Laboratory of Diabetes Mellitus, Shanghai Jiao Tong University Affiliated Sixth People's Hospital.

Results: We found 363 suspected cases by primary screening of haematological variables and haemoglobin analysis. After further genetic testing, four types of $\alpha$ - and $\beta$-thalassaemia mutation were detected in 203 out of 363 individuals. Both $\alpha^{0}$ - and $\alpha^{+}$-thalassaemia mutations, _ $-{ }^{\mathrm{SEA}}$ and $-\alpha^{3.7}$, were identified. $\beta$ Thalassaemia mutations included CD17 (HBB:C.52A>T) and CD26 (HbE or HBB:c.79G $>A$ ). In addition, $13 \mathrm{HbE}$ carriers had coexisting $\alpha^{0}$ - or $\alpha^{+}$-thalassaemia deletions. Clinical haematological variables indicated that, in this study, carriers of all thalassaemic genotypes had more severe hypochromic microcytic anaemia than non-thalassaemic individuals.

Conclusions: Our results provide information on the Jino ethnic minority that may be useful for further genetic counselling, prenatal screening and clinical diagnosis of thalassaemia in this region.

\section{INTRODUCTION}

As a group of monogenic disorders, thalassaemia is a serious health problem worldwide, especially in Mediterranean areas, Southeast Asia and Southern China. ${ }^{1-3}$ Yunnan Province, which is located along the border areas of China-Myanmar-Laos, is notable for its ethnic diversity. According to a previous study of children under 10 years

\section{Strengths and limitations of this study}

- As Jino, the last ethnic minority confirmed in China, was reported to have a high prevalence of thalassaemia according to previous research on children under 10 years of age, this study aimed to detect mutations of $\alpha$ - and $\beta$-thalassaemia in Jino adults.

- The $\alpha$ - and $\beta$-thalassaemia mutation spectrum shown in this research may help to explain further genotype-phenotype correlations and to establish a thalassaemia-prevention programme in this area.

- The sample size we used in genetic testing was relatively small and may not have the validity to identify the rare thalassaemias in this ethnic group.

of age, several ethnic minorities in this region have a high prevalence of thalassaemia, with the prevalence of $\alpha$-thalassaemia ( $\alpha$-thal) being highest $(22.1 \%)$ in Dai from Xishuangbanna and the prevalence of $\beta$-thalassaemia ( $\beta$-thal) being highest in Achang (40.6\%). ${ }^{4}$

Jino is the last ethnic minority confirmed in China, and the prevalence of $\alpha$-thal and $\beta$-thal among Jino children are $3.1 \%$ and $29.3 \%$, respectively. Thalassaemic children may exhibit various clinical symptoms; some are asymptomatic carriers, whereas others have severe haemolytic anaemia. ${ }^{5}$ Blood transfusion therapy, which is needed for severe carriers, imposes a heavy burden on families and public health management. ${ }^{6}$ Although genetic screening is essential to prevent and control this inherited disease, systematic investigations of thalassaemia mutations in Jino adults are rare.

The Jino population comprises nearly 20000 individuals, and most ( $90 \%)$ live around Jino Mountain, which is located in East-Central Yunnan Province. ${ }^{7}$ A large number of thalassaemic mutations have been found in the general population worldwide; ${ }^{8-10}$ however, 
little is known about this isolated population. Indeed, the molecular mechanism and genetic variations of thalassaemia in Jino individuals may be different from those in other ethnicities. Our study aimed to detect $\alpha$-thal and $\beta$-thal gene mutations in Jino adults to provide basic information for further prenatal consulting and thalassaemia diagnosis.

\section{MATERIALS AND METHODS}

\section{Participants and clinical screening}

Ethics approval for the study was granted according to the Declaration of Helsinki (paragraph II) by the institutional review board of Shanghai Jiao Tong University affiliated with the Sixth People's Hospital, Shanghai, China. This cross-sectional study was conducted between February 2012 and April 2012 in eight villages (Luote, Jiama, Balai, Situ, New Situ, Baka, Baya, Dapingzhang) around Jino Mountain in Jinghong, Southern Yunnan Province, China (figure 1). A list of Jino adults from these eight villages was obtained from local villager committee offices. Participants were sampled by a simple computer programme of randomisation from these villages. Staff at the local health centre, who understand both Chinese and Jino languages, contacted the subjects and introduced the purpose of the study. Oral and written informed consent was obtained from all the individuals. Basic demographic information and fasting venous blood samples were collected by researchers.
A total of 1613 Jino adults, including 762 men and 851 women, participated in this survey (haematological and demographic characteristics of the total population included in the study are given in table 1). The following haematological variables were measured: haemoglobin $(\mathrm{Hb})$, mean corpuscular volume $(\mathrm{MCV})$, mean corpuscular haemoglobin $(\mathrm{MCH})$, red blood cell (RBC) and red cell distribution width (RDW). Haemoglobin was analysed by high-performance liquid chromatography (HPLC) using the Variant II haemoglobin analysing system (Bio-Rad Laboratories, Hercules, California, USA). $\alpha$ - and $\beta$-globin genetic testing was performed in participants $(\mathrm{n}=363)$ with hypochromic microcytic anaemia (MCV $<80 \mathrm{fL}$ and/or $\mathrm{MCH}<27 \mathrm{pg}$ ) and/or positive HPLC profiles. In order to evaluate the validity of primary screening approaches for detection of thalassaemia carriers, we randomly selected some of the individuals with negative screening results $(n=50)$ from the remaining participants $(n=1250)$ for further genetic testing.

\section{Genetic testing}

Genomic DNA was extracted from venous blood leucocytes. Three methods were used to detect thalassaemic mutations.

A CapitalBio Thalassaemia Gene Mutation Detection Kit (CapitalBio, Beijing, China) was used to determine 25 common mutations in globin genes in the Chinese population via DNA microarray. Six $\alpha$-thal gene

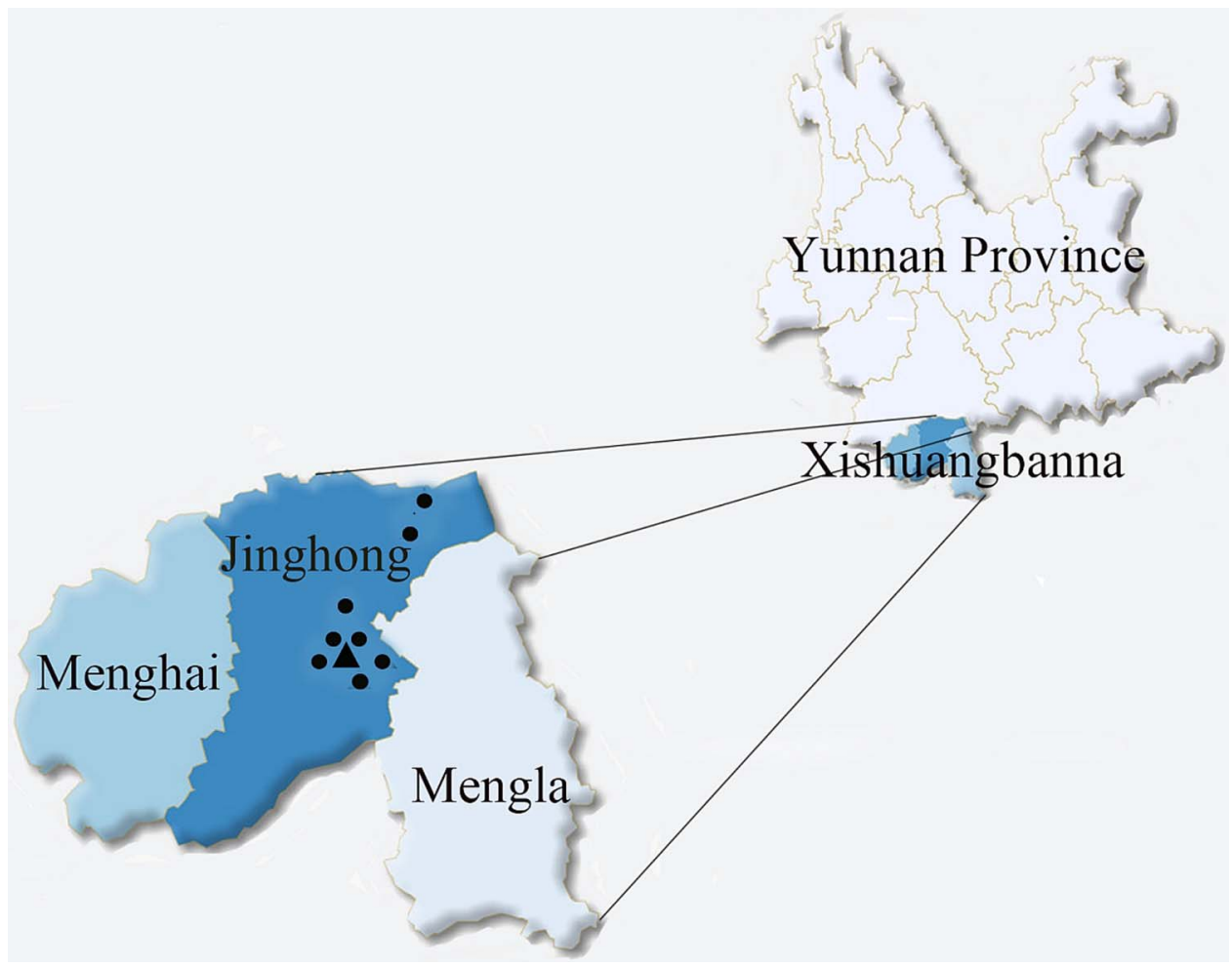

Figure 1 Geographical location of the Jino ethnic minority populations in Yunnan Province, southwest China. The solid black triangle represents Jino Mountain. The solid black circles represent the eight villages in Jinghong where the 1613 subjects were randomly selected. 
Table 1 Haematological and demographic characteristics of 1613 Jino ethnic minority adults included in the study

\begin{tabular}{lccc}
\hline Variable & Total & \multicolumn{1}{l}{ Male } & \multicolumn{1}{l}{ Female } \\
\hline Samples $(n)$ & \multicolumn{1}{c}{1613} & \multicolumn{1}{c}{762} & \multicolumn{1}{c}{851} \\
Age (years) & $40.43 \pm 14.78$ & $40.11 \pm 15.21$ & $40.71 \pm 14.38$ \\
BMl (kg/m $\left.{ }^{2}\right)$ & $21.79 \pm 3.20$ & $22.12 \pm 3.17$ & $21.50 \pm 3.21$ \\
RBC (10 $/ 2 / L)$ & $4.85 \pm 0.56$ & $5.08 \pm 0.57$ & $4.65 \pm 0.47$ \\
RDW (\%) & $12.76 \pm 1.29$ & $12.60 \pm 1.10$ & $12.89 \pm 1.42$ \\
MCV (fL) & $83.42 \pm 8.08$ & $84.97 \pm 7.54$ & $82.03 \pm 8.30$ \\
MCH (pg) & $29.21 \pm 3.26$ & $29.91 \pm 3.07$ & $28.59 \pm 3.30$ \\
HCT (\%) & $40.25 \pm 4.36$ & $42.88 \pm 3.74$ & $37.90 \pm 3.44$ \\
Hb (g/dL) & $14.09 \pm 1.70$ & $15.09 \pm 1.49$ & $13.20 \pm 1.34$
\end{tabular}

Data are shown as mean \pm SD.

$\mathrm{BMI}$, body mass index; $\mathrm{HCT}$, haematocrit; $\mathrm{Hb}$, haemoglobin;

$\mathrm{MCH}$, mean corpuscular haemoglobin; MCV, mean corpuscular

volume; RBC, red blood cell; RDW, red cell distribution width.

mutations and $19 \beta$-thal gene mutations were included. Among them, there were three $\alpha$-thal deletions-that is, the Southeast Asian deletion $\left(-{ }^{\mathrm{SEA}}\right)$, rightward deletion $\left(-\alpha^{3.7}\right)$ and leftward deletion $\left(-\alpha^{4.2}\right)$-and three nondeletional $\alpha$-thal mutations-that is, Hb Constant Spring (HBA2:c.427T >C), Hb Quong Sze (HBA2:c.377T $>$ C or HBA1) and Hb Westmead (HBA2:c.369C>G). Nineteen $\beta$-thal gene mutations were CD14/15 (HBB:c.45 46insG), CD27/28 (HBB:c.84_85insG), CD41/42 (HBB: c.126_129delTCTT), CD71/72 (HBB:c.216_217insA), -32 (HBB:c.-82T>C), -30 (HBB:c.-80T>C), -29 (HBB: c. $-79 \mathrm{~A}>\mathrm{G}),-28$ (HBB:c.-78A $>\mathrm{G}), \mathrm{CD} 17$ (HBB:c.52A $>\mathrm{T})$, CD26 (HBB:c.79G>A), CD30 (HBB:c.91A >G), CD37 (HBB:c.113G>A), CD43 (HBB:c.130G>T), IVS1-1 (HBB: c.92+1G>T), IVS1-5 (HBB:c.92+5G>T), IVS2-5 (HBB: c.315+5G>C), IVS2-654 (HBB:c.316-197C>T), Int (HBB: c.2T>G), CAP (HBB:c.-11_-8delAAAC). A BioMixer II Microarray Hybridisation Station (CapitalBio) was used for hybridisation after multiplex PCR amplification. Then, chips were scanned using a LuxScan 10K-B Microarray Scanner (CapitalBio).

To validate $\beta$-thal mutations, three fragments of the $\beta$-globin gene were amplified. The first fragment was amplified with 5'-CCT AAG CCA GTG CCA GAA GAG C-3' as the forward primer and 5'-TGC CCA GTT TCT ATT GGT CTC $\mathrm{C}_{-} 3^{\prime}$ as the reverse primer, the second fragment was amplified with 5'-TAG AAA CTG GGC
ATG TGG AG-3' as the forward primer and 5'-TGT ACG CTG TTA CTT ATC CC-3' as the reverse primer, and the third fragment was amplified with 5'-TCA GGG CAA TAA TGA TAC AA- $3^{\prime}$ as the forward primer and $5^{\prime}$-TTA GTA GTT GGA CTT AGG GA-3' as the reverse primer. The fragments were sequenced using a 3500 Genetic Analyser (Applied Biosystems, Foster City, California, USA).

We also confirmed three $\alpha$-thal deletions-that is, the Southeast Asian deletion $\left({ }^{\mathrm{SEA}}\right)$, rightward deletion $\left(-\alpha^{3.7}\right)$ and leftward deletion $\left(-\alpha^{4.2}\right)$ - using multiplex gap-PCR assays. Primers and PCR conditions were designed as described in the literature. ${ }^{11} 12$

\section{Statistical analysis}

A statistical analysis was carried out using SAS for Windows (V.9.2). All quantitative traits were tested for normality, and skewed quantitative traits were logarithmically transformed to approximate univariate normality. Data are shown as means \pm SD. Quantitative traits (RBC, Hb, MCV, MCH, RDW) were compared between two groups using the Wilcoxon test, and analysis of variance was performed to compare the differences in the three subgroups of thalassaemia carriers $(\alpha$-Thal, $\beta$-Thal and $\alpha \beta$-Thal). Two-tailed statistical significance was considered at $\mathrm{p}<0.05$.

\section{RESULTS}

\section{Mutations identified in Jino}

Owing to mutations in different globin genes, we observed three groups of thalassaemic carriers, including individuals with only $\alpha$-thal gene deletions or $\beta$-thal gene mutations and individuals with combined $\alpha \beta$-thalassaemia ( $\alpha \beta$-thal) gene mutations. Four different thalassaemia mutations were detected in 203 individuals among 363 suspected cases. No mutations were observed in 50 individuals with negative primary screening results. Table 2 shows the allele frequency of $\alpha$ - and $\beta$-thal mutations found in our study.

\section{Mutations in the $\alpha$-thal gene}

None of the three common non-deletional $\alpha^{+}$-thal mutations, Hb Constant Spring (HBA2:c.427T>C), Hb Quong

Table 2 Allele frequency of $\alpha$ - and $\beta$-thalassaemia mutations found in our study

\begin{tabular}{|c|c|c|c|c|}
\hline Mutation & Phenotype & $\mathbf{n}$ & Number of alleles & Allele frequency (ratio) \\
\hline \multicolumn{5}{|c|}{$\alpha$-Thalassaemia } \\
\hline --SEA & $\alpha \% \alpha$ & 42 & 42 & $70.00 \%(42 / 60)$ \\
\hline$-\alpha^{3.7}$ & $\alpha^{+} / \alpha$ & 16 & 16 & $30.00 \%(18 / 60)$ \\
\hline$-\alpha^{3.7} /-\alpha^{3.7}$ & $\alpha^{+} / \alpha^{+}$ & 1 & 2 & - \\
\hline \multicolumn{5}{|c|}{$\beta$-Thalassaemia } \\
\hline $\mathrm{CD} 17$ & $\beta^{0} / \beta^{A}$ & 20 & 20 & $12.35 \%(20 / 162)$ \\
\hline $\mathrm{HbE}$ & $\beta^{\mathrm{E}} / \beta^{\mathrm{A}}$ & 132 & 132 & $87.65 \%(142 / 162)$ \\
\hline $\mathrm{HbE} / \mathrm{HbE}$ & $\beta^{\mathrm{E}} / \beta^{\mathrm{E}}$ & 5 & 10 & - \\
\hline
\end{tabular}


Figure 2 Gel electrophoresis of PCR amplifying results in $\alpha$-thal deletions. M, marker, 200 bp DNA Ladder; lane 1, rightward deletion (genotype of $-\alpha^{3.7} /-\alpha^{3.7}$ ); lanes 2 and 3 , rightward deletion (genotype of $-\alpha^{3.7} / \alpha \alpha$ ); lanes 4 and 5 , Southeast Asia deletion (genotype of.$--\mathrm{SEA} / \alpha \alpha$ ).

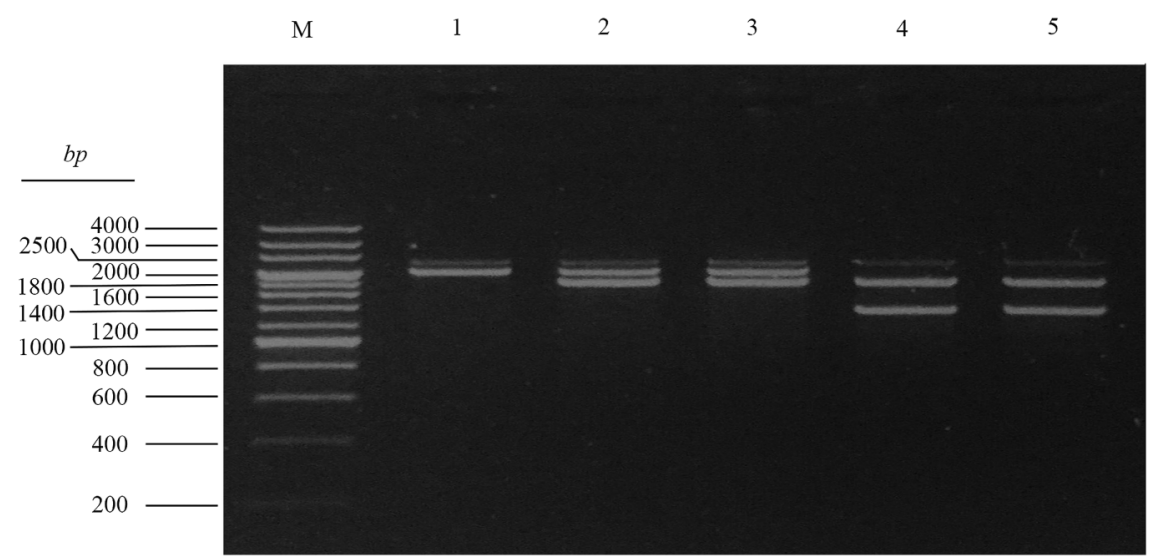

M

Sze (HBA2:c.377T>C (or HBA1)) and Hb Westmead (HBA2:c.369C>G), were found in 203 participants with thalassaemia mutations. Forty-six of 203 participants carried $\alpha$-thal deletions only; _SEA and $-\alpha^{3.7}$ were observed, accounting for $16.7 \%(34 / 203)$ and $5.9 \%$ $(12 / 203)$ of the mutations, respectively. Among these individuals, we identified both $\alpha^{+} / \alpha$ and $\alpha^{+} / \alpha^{+}$for $-\alpha^{3.7}$ and $\alpha^{0} / \alpha$ for ${ }_{-}$SEA (gel electrophoresis of PCR amplifying results are shown in figure 2). However, no $-\alpha^{4.2}$ deletion was observed.

\section{Mutations in the $\beta$-thal gene}

We observed mutations in CD17 (HBB:c.52A>T) (figure 3) and $\mathrm{CD} 26$ ( $\mathrm{HbE}$ or $\mathrm{HBB}: \mathrm{c} .79 \mathrm{G}>\mathrm{A}$ ) (figure 4). CD17, which accounted for $9.9 \%(20 / 203)$ of mutations, was found to be $\beta^{0} / \beta^{\mathrm{A}}$ in this population. Participants with $\mathrm{HbE}$ variant only, either $\beta^{\mathrm{E}} / \beta^{\mathrm{A}}$ or $\beta^{\mathrm{E}} / \beta^{\mathrm{E}}$, accounted for $61.1 \%(124 / 203)$ of mutations. Furthermore, $13 \mathrm{HbE}$ carriers harboured -SEA $(n=8)$ or $-\alpha^{3.7}(n=5)$ at a combined frequency of $6.4 \%(13 / 203)$.

\section{Haematological features of different thalassaemia genotypes}

The haematological data of different thalassaemia genotypes are summarised in table 3. Compared with normal individuals, thalassaemic carriers had significantly lower $\mathrm{Hb}, \mathrm{MCV}$ and MCH levels $(\mathrm{p}<0.001$, respectively) and higher RBC and RDW levels $(p<0.001$, respectively).
Figure 3 Heterozygous CD17 $(A>T)$ mutation $(A)$ and the corresponding normal sequence of $\beta$-globin. Red arrow indicates the position of this point mutation.

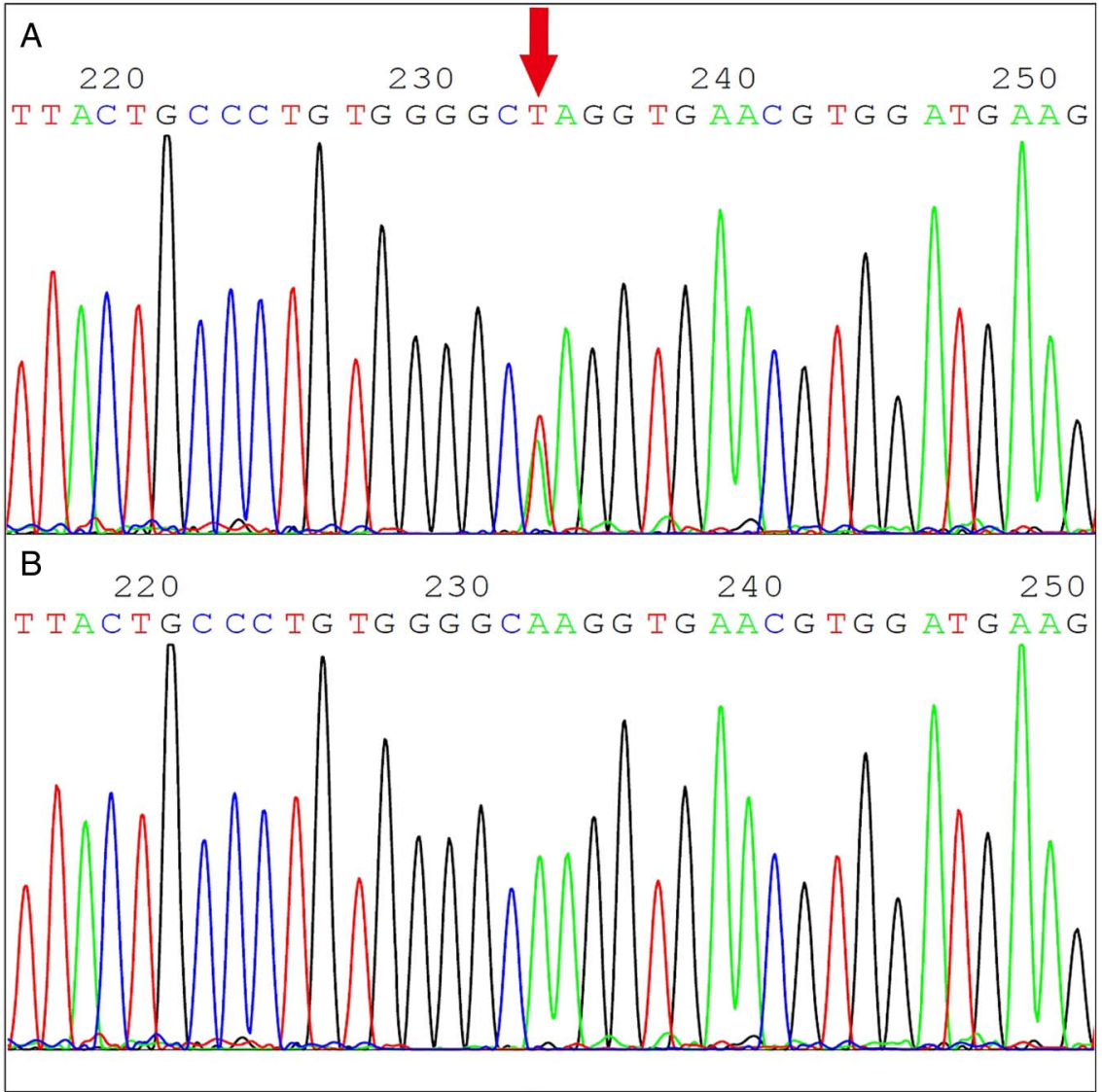


Figure 4 Heterozygous CD26 $(G>A)$ mutation $(A)$ and the corresponding normal sequence of $\beta$-globin. Red arrow indicates the position of this point mutation.

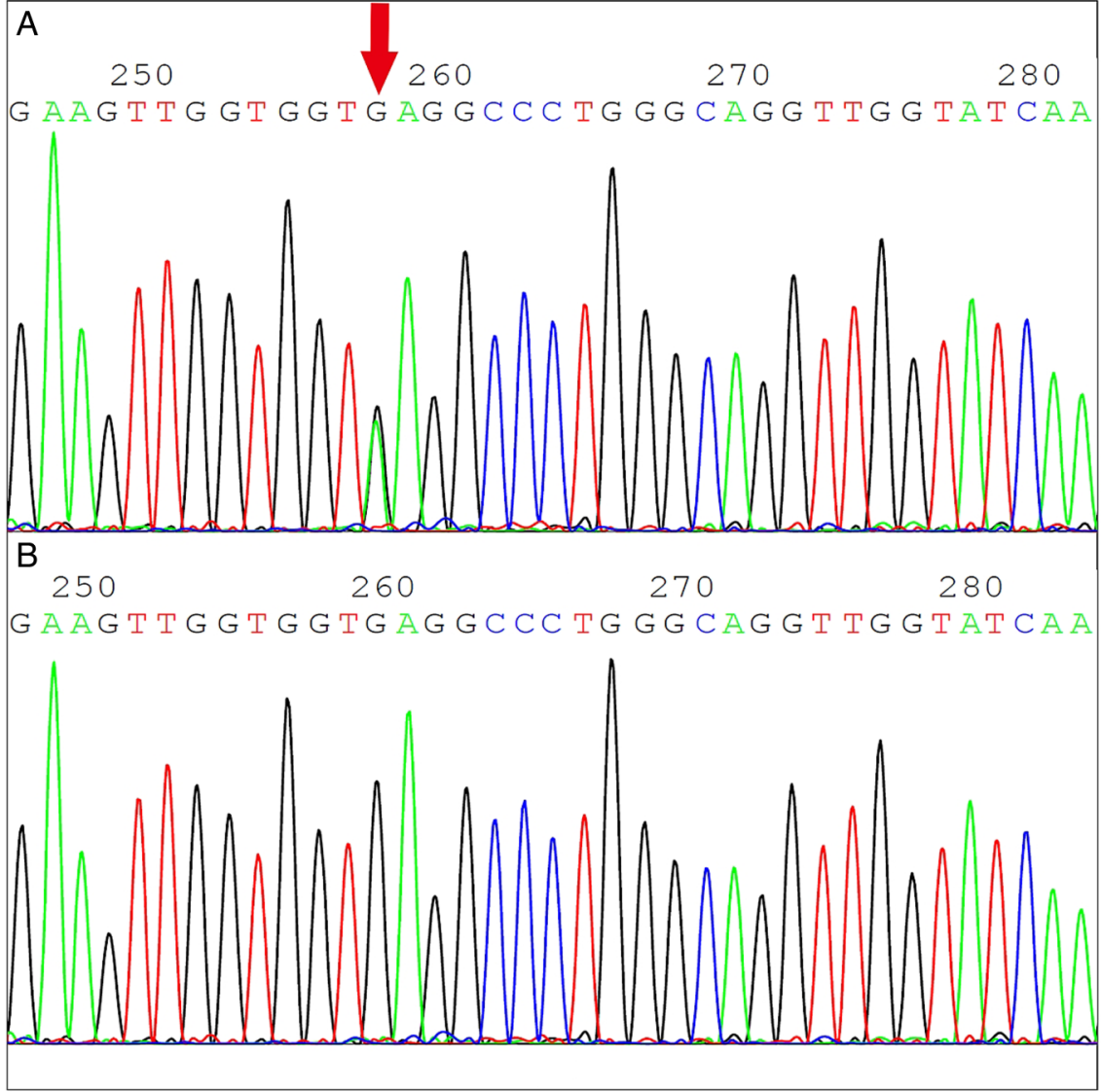

Furthermore, we compared the differences in these five indexes among the three groups of carriers ( $\alpha$-thal, $\beta$-thal and $\alpha \beta$-thal). Significant differences in MCV $(\mathrm{p}=0.0111)$, MCH $(\mathrm{p}=0.0002)$ and RBC $(\mathrm{p}=0.0012)$ were observed between these groups. MCV and MCH levels in the $\alpha$-thal group were significantly lower than those in the $\beta$-thal group $(p<0.05)$, whereas $R B C$ levels were higher $(\mathrm{p}<0.05)$. In contrast, no difference was observed between the $\alpha \beta$-thal and $\alpha$-thal groups $/ \beta$-thal groups. Moreover, there was a tendency towards increased RDW

Table 3 Haematological data of 1613 Jino ethnic minority individuals with different thalassaemia subtypes

\begin{tabular}{|c|c|c|c|c|c|c|}
\hline Thalassaemia type & $\mathbf{n}$ & $\operatorname{RBC}\left(10^{12} / L\right)$ & $\mathrm{Hb}(\mathrm{g} / \mathrm{dL})$ & $\operatorname{MCV}(f L)$ & $\mathrm{MCH}(\mathrm{pg})$ & RDW (\%) \\
\hline$\alpha$-Thalassaemia & 46 & $5.63 \pm 0.78$ & $13.03 \pm 1.61$ & $68.99 \pm 6.50$ & $23.33 \pm 2.45$ & $13.64 \pm 1.38$ \\
\hline$\alpha / \alpha$ & 34 & $5.88 \pm 0.68$ & $12.98 \pm 1.62$ & $65.69 \pm 2.65$ & $22.08 \pm 0.81$ & $14.01 \pm 1.37$ \\
\hline$\alpha^{+} / \alpha$ & 11 & $4.86 \pm 0.58$ & $13.19 \pm 1.70$ & $78.96 \pm 4.43$ & $27.14 \pm 1.87$ & $12.52 \pm 0.74$ \\
\hline$\alpha^{+} / \alpha^{+}$ & 1 & 5.34 & 12.7 & 71.5 & 23.8 & 13.1 \\
\hline$\beta$-Thalassaemia & 144 & $5.27 \pm 0.48$ & $13.31 \pm 1.70$ & $72.46 \pm 7.09$ & $25.29 \pm 2.75$ & $13.21 \pm 0.96$ \\
\hline$\beta^{0} / \beta^{\mathrm{A}}$ & 20 & $5.41 \pm 0.38$ & $10.68 \pm 0.53$ & $58.70 \pm 2.31$ & $19.77 \pm 0.83$ & $14.23 \pm 0.40$ \\
\hline$\beta^{\mathrm{E}} / \beta^{\mathrm{A}}$ & 120 & $5.23 \pm 0.48$ & $13.77 \pm 1.41$ & $75.17 \pm 3.88$ & $26.34 \pm 1.49$ & $12.98 \pm 0.85$ \\
\hline$\beta^{\mathrm{E}} / \beta^{\mathrm{E}}$ & 4 & $5.91 \pm 0.51$ & $12.55 \pm 1.22$ & $60.23 \pm 3.61$ & $21.23 \pm 1.15$ & $15.00 \pm 0.57$ \\
\hline$\alpha \beta$-Thalassaemia & 13 & $5.67 \pm 0.70$ & $13.63 \pm 1.67$ & $69.40 \pm 6.85$ & $24.17 \pm 2.59$ & $13.12 \pm 0.92$ \\
\hline$\beta^{\mathrm{E}} / \beta^{\mathrm{A}}$ with $\alpha^{\mathrm{O}} / \alpha$ & 7 & $6.02 \pm 0.69$ & $13.57 \pm 1.58$ & $65.94 \pm 2.82$ & $22.57 \pm 1.07$ & $13.66 \pm 0.71$ \\
\hline$\beta^{\mathrm{E}} / \beta^{\mathrm{A}}$ with $\alpha^{+} / \alpha$ & 5 & $5.21 \pm 0.53$ & $14.08 \pm 1.88$ & $76.46 \pm 3.86$ & $27.00 \pm 1.33$ & $12.22 \pm 0.36$ \\
\hline$\beta^{\mathrm{E}} / \beta^{\mathrm{E}}$ with $\beta^{\mathrm{O}} / \beta^{\mathrm{A}}$ & 1 & 5.57 & 13.8 & 58.3 & 21.2 & 11.8 \\
\hline Total thalassaemia & 203 & $5.38 \pm 0.60$ & $13.27 \pm 1.68$ & $71.48 \pm 7.08$ & $24.77 \pm 2.79$ & $13.30 \pm 1.08$ \\
\hline Non-thalassaemia & 1410 & $4.78 \pm 0.51$ & $14.21 \pm 1.67$ & $85.14 \pm 6.64$ & $29.85 \pm 2.79$ & $12.68 \pm 1.30$ \\
\hline p Value ${ }^{*}$ & & $<0.001$ & $<0.001$ & $<0.001$ & $<0.001$ & $<0.001$ \\
\hline $\mathrm{p}$ Value $\dagger$ & & 0.0012 & $>0.05$ & 0.0111 & 0.0002 & 0.0573 \\
\hline
\end{tabular}

Data are shown as $n$, mean $\pm S D$, or raw data when necessary.

*Non-thalassaemic individuals compared with thalassaemia group.

†Compared among three subgroups of thalassaemia ( $\alpha$-Thal, $\beta$-Thal and $\alpha \beta$-Thal).

$\mathrm{Hb}$, haemoglobin; $\mathrm{MCH}$, mean corpuscular haemoglobin; MCV, mean corpuscular volume; RBC, red blood cell; RDW, red cell distribution width. 
levels in the $\alpha$-thal group compared with the $\beta$-thal group $(\mathrm{p}=0.0573)$.

\section{DISCUSSION}

Thalassaemia is a common monogenic disease with a relatively high prevalence in Southeast Asia. In China, this disease is mainly prevalent in areas near the southern bank of the Yangtze River, such as Guangdong, Guangxi, Fujian and Yunnan Provinces. ${ }^{13-15}$ Prenatal screening and related molecular diagnoses are crucial for preventing and treating thalassaemia. Many thalassaemia studies have been conducted in Yunnan Province. ${ }^{1617}$ However, data on the Jino population are limited because this population is the last ethnic minority confirmed in China.

We randomly selected 1613 Jino adults from eight villages around Jino Mountain in Jinghong, Southern Yunnan. Among the gene mutations identified, the most prevalent $\alpha$-thal and $\beta$-thal genotypes in this region were _SEA and HbE, in agreement with previous data from Yunnan Province. ${ }^{18} 19$ According to our results, the overall prevalence of thalassaemia in Jino was nearly $12.6 \%$, which is similar to the prevalence observed in Kunming. ${ }^{20}$ Prevalence of $\alpha \beta$-thal $(8 \%)$ in our population was equal to that in the $\mathrm{Li}$ population in Hainan Province $(7.99 \%)$, where thalassaemia prevalence is high. $^{21}$ Although Yunnan Province has a high prevalence of thalassaemia with diverse genotypes, the spectrum of globin gene mutations among the Jino population is relatively limited.

HbE, a type of haemoglobinopathy, can be observed in most regions of Southeast China. ${ }^{22}$ Due to a point mutation in the $\beta$-globin gene, the balance of various globin products is disrupted, leading to a structural haemoglobin variant. Although $\mathrm{HbE}$ carriers may only have slight anaemia, their offspring will exhibit severe clinical symptoms in the presence of other $\beta$-thal types. ${ }^{23}$ Therefore, potential $\mathrm{HbE}$ carriers should undergo genetic testing and prenatal counselling. HPLC is often used as an efficient primary screening method to detect abnormal $\mathrm{Hb}$, as was carried out in this study and previous studies. $^{2425}$ In our study, 95.6\% (131/137) of $\mathrm{HbE}$ carriers were identified by HPLC, and 13 of these individuals had concomitant $\alpha$-thal deletions.

Different genotypes lead to different clinical phenotypes. ${ }^{26}$ We found that thalassaemic carriers had significantly lower MCV and MCH levels. Regarding those with $\beta$-thal mutations, MCV and MCH levels were significantly decreased in CD17 carriers compared with HbE carriers, suggesting that a nonsense mutation in the $\beta$-globin gene causes greater erythrocyte impairment. Hypochromic microcytic anaemia was moderate in individuals with $\beta^{\mathrm{E}} / \beta^{\mathrm{A}}$ and $\alpha^{+} / \alpha$ compared with $\beta^{\mathrm{E}} / \beta^{\mathrm{A}}$ carriers. This paradox may be explained by the fact that changes in the $\alpha$ - and $\beta$-globin chains may balance each other out when both mutations coexist in an individual. Accordingly, rapidly estimating the genetic state of an illness based on haematological variables is difficult. Therefore, genetic screening of both $\alpha$ - and $\beta$-globin gene mutations in potential parents is of utmost importance to prevent births with severe defects. ${ }^{27}$

However, there are some limitations in this study. First, the sample size we used in the genetic testing was relatively small and may not have the validity to identify the rare thalassaemia variants from this ethnic group. Second, investigations of population and family structure were not performed in this study, although $\alpha$-thal and $\beta$-thal gene mutations were common among the Jino ethnic minority. As a result, further studies on the inbreeding levels and consanguinity structure are warranted to reveal the underlying mechanism of gene flow and then assess the occurrence and persistence of $\alpha$-thal and $\beta$-thal gene mutations, especially coexisting $\alpha$-thal and $\beta$-thal gene mutations within Jino individuals.

In conclusion, this study revealed $\alpha$ - and $\beta$-thal mutations in the Jino ethnic minority population in Yunnan Province. Of these mutations, _- ${ }^{\mathrm{SEA}}$ and $\mathrm{HbE}$ were the most prevalent $\alpha$-thal and $\beta$-thal gene mutation types, respectively. In addition, data based on clinical haematological variable analysis indicated that the severity of hypochromic microcytic anaemia is associated with the genotype of thalassaemia. Our results provide evidence that may be useful for further genetic counselling, prenatal screening and clinical diagnosis of thalassaemia in this region.

Acknowledgements We thank all of the participants for their dedication. We acknowledge the skilful technical support of all nursing and medical staff at the Shanghai Clinical Center for Diabetes.

Contributors WJ and $\mathrm{CH}$ conceived and designed the experiments. SW and RZ performed the experiments and analysed the data. GX, YL, XH, FuJ and FeJ contributed materials and analysis tools. SW prepared the article. $\mathrm{CH}$ and WJ revised the manuscript. All the authors have read and approved the final version of this manuscript.

Funding This work was financially supported by a grant from the National Natural Science Foundation of China (91331110).

Competing interests None declared.

Patient consent Obtained.

Ethics approval According to the Declaration of Helsinki (paragraph II), ethics approval for the study was granted by the institutional review board of Shanghai Jiao Tong University affiliated with the Sixth People's Hospital, Shanghai, China. Written and Oral informed consent was obtained from all participants.

Provenance and peer review Not commissioned; externally peer reviewed.

Data sharing statement No additional data are available.

Open Access This is an Open Access article distributed in accordance with the Creative Commons Attribution Non Commercial (CC BY-NC 4.0) license, which permits others to distribute, remix, adapt, build upon this work noncommercially, and license their derivative works on different terms, provided the original work is properly cited and the use is non-commercial. See: http:// creativecommons.org/licenses/by-nc/4.0/

\section{REFERENCES}

1. Weatherall DJ. Thalassemia as a global health problem: recent progress toward its control in the developing countries. Ann $N Y$ Acad Sci 2010;1202:17-23. 
2. Fucharoen $S$, Winichagoon $P$. Thalassemia in SouthEast Asia: problems and strategy for prevention and control. Southeast Asian $J$ Trop Med Public Health 1992;23:647-55.

3. Li B, Zhang XZ, Yin AH, et al. High prevalence of thalassemia in migrant populations in Guangdong Province, China. BMC Public Health 2014;14:905.

4. Yao LQ, Zou TB, Yang FB, et al. [Epidemiological study of thalasaemia among children in Xishuangbanna, Dehong and Nujiang of Yunnan province]. Zhonghua Yi Xue Yi Chuan Xue Za Zhi 2011;28:579-82.

5. Sankaran VG, Weiss MJ. Anemia: progress in molecular mechanisms and therapies. Nat Med 2015;21:221-30.

6. Goss C, Giardina P, Degtyaryova D, et al. Red blood cell transfusions for thalassemia: results of a survey assessing current practice and proposal of evidence-based guidelines. Transfusion 2014;54:1773-81.

7. Xu JW, Liao YM, Liu H, et al. Use of bed nets and factors that influence bed net use among Jinuo Ethnic Minority in southern China. PLOS ONE 2014;9:e103780.

8. Piel FB, Weatherall DJ. The $\alpha$-thalassemias. $N$ Engl $J$ Med 2014:371:1908-16.

9. Rund D, Rachmilewitz E. Beta-thalassemia. N Engl J Med 2005;353:1135-46.

10. Saller E, Dutly F, Frischknecht $\mathrm{H}$. Two Novel alpha2 Gene Mutations Causing Altered Amino Acid Sequences Produce a Mild $(\mathrm{Hb}$ Kinshasa, HBA2: c.428A > T) and Severe (HBA2: c.342-345insCC) alpha-Thalassemia Phenotype. Hemoglobin 2015:1-3.

11. Zhou Y, Zhang Y, Li L, et al. [Rapid detection of three common deletional alpha thalassemias in Chinese by single-tube multiplex PCR]. Zhonghua Yi Xue Yi Chuan Xue Za Zhi 2005;22:180-4.

12. Chong SS, Boehm CD, Higgs DR, et al. Single-tube multiplex-PCR screen for common deletional determinants of alpha-thalassemia. Blood 2000;95:360-2.

13. Xiong $\mathrm{F}$, Sun $\mathrm{M}$, Zhang $\mathrm{X}$, et al. Molecular epidemiological survey of haemoglobinopathies in the Guangxi Zhuang Autonomous Region of southern China. Clin Genet 2010;78:139-48.

14. Yin A, Li B, Luo M, et al. The prevalence and molecular spectrum of $\alpha$ - and $\beta$-globin gene mutations in 14,332 families of Guangdong Province, China. PLoS ONE 2014;9:e89855.
15. Huang $\mathrm{H}, \mathrm{Xu} \mathrm{L}$, Lin $\mathrm{N}$, et al. Molecular spectrum of $\beta$-thalassemia in Fujian Province, Southeastern China. Hemoglobin 2013;37:343-50.

16. Zhu BS, He J, Zhang J, et al. [A study on gene mutation spectrums of $\alpha$ - and $\beta$-thalassemias in populations of Yunnan Province and the prenatal gene diagnosis]. Zhonghua Fu Chan Ke Za Zhi 2012;47:85-9.

17. Zou T, Yao L, Li Q, et al. The family-based research and genetic diagnosis of $\beta$-thal major in Dai ethnic. Zhonghua Xue Ye Xue Za Zhi 2014;35:260-1.

18. Zhang J, He J, Zeng $\mathrm{XH}$, et al. Genetic heterogeneity of the $\beta$-globin gene in various geographic populations of Yunnan in Southwestern China. PLOS ONE 2015;10:e0122956.

19. Zhang J, Zhu BS, He J, et al. The spectrum of $\alpha$ - and $\beta$-thalassemia mutations in Yunnan Province of Southwestern China. Hemoglobin 2012;36:464-73.

20. Wen BP, Fan M, Dai HJ, et al. Biochemical screening and genetic diagnosis of thalassemia in children from Kunming. Zhongguo Dang Dai Er Ke Za Zhi 2011;13:104-6.

21. Yao H, Chen X, Lin L, et al. The spectrum of $\alpha$ - and $\beta$-thalassemia mutations of the Li people in Hainan Province of China. Blood Cells Mol Dis 2014:53:16-20.

22. Chen $\mathrm{W}$, Zhang $X$, Shang $X$, et al. The molecular basis of beta-thalassemia intermedia in southern China: genotypic heterogeneity and phenotypic diversity. BMC Med Genet 2010;11:31

23. Li YQ, Huang HP, Qin GF, et al. [Phenotype and genotype analysis of hemoglobin E]. Zhonghua Xue Ye Xue Za Zhi 2012;33:861-4.

24. Eastman JW, Wong R, Liao CL, et al. Automated HPLC screening of newborns for sickle cell anemia and other hemoglobinopathies. Clin Chem 1996;42:704-10.

25. Khera R, Singh T, Khuana N, et al. HPLC in characterization of hemoglobin profile in thalassemia syndromes and hemoglobinopathies: a clinicohematological correlation. Indian $J$ Hematol Blood Transfus 2015;31:110-15.

26. Bozdogan ST, Yuregir OO, Buyukkurt N, et al. Alpha-thalassemia mutations in Adana Province, southern Turkey: genotype-phenotype correlation. Indian J Hematol Blood Transfus 2015;31:223-8.

27. Cao A, Kan YW. The prevention of thalassemia. Cold Spring Harb Perspect Med 2013;3:a011775. 